\title{
Mini Track: 'Evaluation of implementation, adoption and diffusion of IS in Healthcare (HCADI)
}

\author{
Mini Track Chairs: \\ Roel Schuring \& Ton Spil \\ University of Twente, The Netherlands
}

Developing, implementing and using information technology in organisations is a complex social activity. It is often characterised by ill-defined problems or vague goals, conflicts and disruptions that result from organisational change. Successfully implementing information systems in healthcare organisations appears to be a difficult task. Information Technology is seen as an enabler of change in healthcare organisations but (information) technology adoption decisions in healthcare are complex because of the uncertainty of benefits and the rate of change of technology. Relevance and micro-relevance are seen as important determinants for IS success. The (lack of) adoption of IT traditionally found it's reasons in resistance of the professionals in healthcare organisations but this is clearly changing in healthcare today. When implementing an information system in healthcare it is wise to focus on the success determinants relevance, resistance, requirements and resources. This new minitrack attracted papers on experiences with implementation of information systems in healthcare. Also more theoretical contributions on evaluation and IS success and adoption are encouragedns arising.

The first contribution by Horan, Tulu, Hilton \& Burton "Use of online systems in clinical medical assessments: an analysis of physician acceptance of online disability evaluation systems" seeks to develop a conceptual model for physician acceptance and test this socio-work structure via a survey. The model is a microapproach as it focusses on work-practice considerations of physicians, factors affecting physicians' acceptance and use of decision support systesm in the clinical setting and tasktechnology fit. The contribution by Gagnon, Lamothe, Fortin, Cloutier, Godin, Gagné and Reinharz; "The impact of Organizational characteristics on telehealth adoption by hospitals" analyses adoption on organisational level. It is structured around hypothesis that are based on previous research and that are tested on the basis of research in 32 healthcare centres involved as telehealth services. The contribution by Maass and Suomi "Adoption-related aspects of an IS system in a health care setting." focusses on adoption of a digital image (PACS) system. It considers particularly financial aspects of this implementation. Finally, Fitch contributed "Information Systems in Healthcare: Mind the Gap". It considers the gap between healthcare professionals and information technology planners. 